\title{
Association of multispecific red blood cell alloimmunization with HLA-Class II variants is related to Rh phenotypes
}

\author{
Maluskova $\mathrm{A}^{1}$, Mrazek $\mathrm{F}^{2}$, Kozelska $\mathrm{R}^{1}$, Koristka $\mathrm{M}^{1}$, Cermakova $\mathrm{Z}^{1}$ \\ Blood Centre, University Hospital, Ostrava, Czech Republic. alena.maluskova@fno.cz
}

\begin{abstract}
AIM: It remains unclear, why only some patients form alloantibodies against foreign RBC antigens. Transfusion of red blood cell (RBC) products and pregnancy are the most relevant causes of immunization against RBC alloantigens. Here we investigated the relationship between RBC alloantibodies, Rh phenotype, and HLA phenotype among patients with multiple RBC alloantibodies

METHODS: In a group of 124 multi-responders - including both pregnant women and transplant recipients - we analysed the distribution of HLA-Class II variants in subgroups of multi-responders to RBC alloantigens according to their Rh status.

RESULTS: As expected, the RhD-negative phenotype was overrepresented in our alloimmunized group $(49.2 \%)$ compared to in the general population. Importantly, HLA-DRB1*15 carriers were significantly overrepresented among D-negative multi-responders compared to D-positive multi-responders $(P c=0.045)$. Furthermore, the linked HLA-DRB1*13, HLA-DQB1*06, and HLA-DQA1*01 variants were more frequent in individuals with the DCCee phenotype than in other RhD-positive phenotypes.

CONCLUSION: Our present findings showed that RBC multispecific alloimmunization was associated with particular HLA-Class II variants based on Rh status (Tab. 3, Ref. 22). Text in PDF www.elis.sk

KEY WORDS: HLA, red blood cell (RBC) antigens and antibodies (Abs), Rh phenotypes, transfusion therapy.
\end{abstract}

\section{Introduction}

Blood product transfusion is an essential and irreplaceable supportive therapy in many diseases. However, even when the donor and recipient are conventionally matched in terms of ABO (D and $\mathrm{K}$ antigens are taken into account strictly only in young girls and in women in the fertile period), red blood cell (RBC) product usage can lead to the formation of alloimmune antibodies against the other RBC alloantigens. Although such RBC alloimmunization is a serious and frequent complication of transfusion therapy (1), only a minority of individuals, who receive RBC transfusion develop alloantibodies (2, 3). RBC alloimmunization incidence rates range from $18-65 \%$ and are significantly higher in patients with sickle cell disease or thalassemia compared to general population (4-8).

It is not yet fully understood, why only some RBC product recipients form alloantibodies against the foreign donor RBC anti-

${ }^{1}$ Blood Centre, University Hospital, Ostrava, Czech Republic, and ${ }^{2}$ Department of Immunology, Faculty of Medicine and Dentistry, Palacky University, Olomouc, Czech Republic

Address for correspondence: A. Maluskova, University Hospital Ostrava, 17. listopadu 1790/5, CZ-708 52 Ostrava - Poruba, Czech Republic Phone: +420.597374421 , Fax: +420.596917340

Acknowledgements: This study was supported by institutional grants from University Hospital Ostrava (RVO-FNOs/2014) and Palacky University Olomouc (IGA_LF_2020_016). gens, but several established risk factors are associated with RBC alloimmunization, including donor and recipient RBC antigen disparity, recipient genetic factors, and inflammatory state. With regards to immunogenetic factors, several studies have demonstrated an association between the presence of single alloantibodies and particular HLA-DRB1 variants (9-13). Moreover, RBC multi-responder status is generally associated with HLA-DRB1*15 variant (13-15), supporting the concept that HLA restriction may play an important role in susceptibility to RBC alloimmunization.

Based on previous findings regarding genetic susceptibility to RBC alloimmunization, in the present study, we focused on patients with multi-specific antibody formation, i.e., "multi-responders”. Here we investigated the relationship between RBC alloantibodies, Rh phenotype, and HLA phenotype within a group of patients with multiple RBC alloantibodies.

\section{Materials and methods}

\section{Study population}

Residual blood samples were collected from RBC-immunized individuals between March 2014 and December 2015. We performed pre-sampling based on antibody specificities from the Blood Centre database of the University Hospital of Ostrava. The study ultimately enrolled 124 patients with more than one RBC alloantibody ("multi-responders"), i.e., with selected combinations of alloantibodies against Rh (D, C, E, c, e), Kell (K), Kidd (Jk', $\left.\mathrm{Jk}^{\mathrm{a}}\right)$, and Duffy $\left(\mathrm{Fy}^{\mathrm{a}}\right)$ antigens. 
Tab. 1. Number $(\mathrm{N})$ of individuals, $\mathrm{F} / \mathrm{M}$ ratio, and $\mathrm{RhD}$ phenotype of the subgroups according to the particular antibody combinations in multi-responders $(n=124)$.

\begin{tabular}{lccccc}
\hline Antibody combination & $\begin{array}{c}\text { Number of } \\
\text { individuals }\end{array}$ & F/M ratio & $\begin{array}{c}\text { Number of } \\
\text { RhD } \\
\text { positive }\end{array}$ & $\begin{array}{c}\text { Number of } \\
\text { RhD } \\
\text { negative }\end{array}$ & $\begin{array}{c}\text { RhCE } \\
\text { phenotypes }\end{array}$ \\
\hline $\mathrm{C}+\mathrm{D}^{*}$ & 46 & 2.8 & 0 & 46 & ccee \\
\hline $\mathrm{C}+\mathrm{D}$ (only 2 Abs) & 33 & 2.6 & 0 & 33 & ccee \\
\hline $\mathrm{E}+$ other Abs & 61 & 2.8 & 44 & 17 & - \\
\hline $\mathrm{E}+\mathrm{C}^{*}$ & 20 & 2.0 & 20 & 0 & CCee \\
\hline $\mathrm{E}+\mathrm{CW}^{*}$ & 14 & 6 & 12 & 2 & $\begin{array}{c}\text { Ccee } \\
\text { CCee }\end{array}$ \\
\hline $\mathrm{E}+\mathrm{D}^{*}$ & 12 & 3 & 0 & 12 & $\begin{array}{c}\text { ccee } \\
\text { Ccee }\end{array}$ \\
\hline $\mathrm{E}+\mathrm{K} / \mathrm{Kp} ; \mathrm{E}+\mathrm{Le}{ }^{\mathrm{a}} ; \mathrm{S}+\mathrm{Jk}^{\mathrm{a}} / \mathrm{Fy}^{\mathrm{a}} / \mathrm{Lu} / \mathrm{M}$ & 11 & 1.75 & 11 & 0 & $\begin{array}{c}\text { Ccee } \\
\text { CCee }\end{array}$ \\
\hline $\mathrm{e}+\mathrm{C}^{*}$ & 8 & 3 & 8 & 0 & ccEE \\
\hline $\mathrm{K}+\mathrm{c}^{*} ; \mathrm{K}+\mathrm{Cw}$ & 4 & 3 & 4 & 0 & CCee
\end{tabular}

$\mathrm{Fy}^{\mathrm{a}}+\mathrm{c}, \mathrm{Fy}^{\mathrm{a}}+\mathrm{Cw}, \mathrm{C}+\mathrm{Jk}^{\mathrm{a}}, \mathrm{M}+\mathrm{Kp}$,

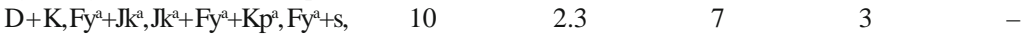

$\mathrm{D}+\mathrm{Le}^{\mathrm{a}}, \mathrm{Cw}+\mathrm{f}, \mathrm{K}+\mathrm{Le}^{\mathrm{a}}, \mathrm{K}+\mathrm{C}+\mathrm{Fy}^{\mathrm{a}}$

\begin{tabular}{llllll}
\hline $\mathrm{C}+\mathrm{G}^{* *}$ & 2 & - & 0 & 2 & ccee \\
\hline
\end{tabular}

* Other specificities could be present. ${ }^{* *}$ Individuals with anti-C+D antibodies were differentiated from those with anti-G antibodies only among pregnant women; these specificities could not be distinguished in transfused patients. Rh phenotypes were collected from the database of the University Hospital of Ostrava.

Tab. 2. Comparisons of phenotypic frequencies of HLA-DRB1, HLA-DQB1, and HLA-DQA1 variants among the $D$-negative $(n=61)$ and $D$-positive $(n=63)$ multi-responders.

\begin{tabular}{|c|c|c|c|c|c|c|}
\hline $\begin{array}{l}\text { Allelic } \\
\text { groups }\end{array}$ & $\begin{array}{l}\text { Percentage of } \\
\text { RhD negative }\end{array}$ & $\begin{array}{l}\text { Percentage of } \\
\text { RhD positive }\end{array}$ & $\mathrm{p}$ & OR & $95 \%$ CI & Pc value $^{a}$ \\
\hline DRB1*01 & 22.9 & 22.2 & 1.0 & & & \\
\hline DRB1*03 & 19.7 & 28.6 & 0.2 & & & \\
\hline DRB1*04 & 11.5 & 22.2 & 0.1 & & & \\
\hline DRB1*07 & 29.5 & 30.2 & 1.0 & & & \\
\hline DRB1*08 & 3.3 & 3.2 & 1.0 & & & \\
\hline DRB1*09 & 3.3 & 0 & 0.8 & & & \\
\hline DRB1*10 & 3.3 & 1.6 & 1.0 & & & \\
\hline DRB1*11 & 21.3 & 20.6 & 1.0 & & & \\
\hline DRB1*12 & 3.3 & 1.6 & 1.0 & & & \\
\hline DRB1*13 & 16.4 & 28.6 & 0.1 & & & \\
\hline DRB1*14 & 1.6 & 6.3 & 0.2 & & & \\
\hline DRB1*15 & 50.8 & 25.4 & 0.0035 & 3.04 & $1.4-6.5$ & 0.045 \\
\hline DRB1*16 & 1.6 & 7.9 & 0.1 & & & \\
\hline DQB1*02 & 42.6 & 46.0 & 0.7 & & & \\
\hline DQB1*03 & 39.3 & 47.6 & 0.4 & & & \\
\hline DQB1*04 & 1.6 & 1.6 & 1.0 & & & \\
\hline DQB1*05 & 32.8 & 39.7 & 0.4 & & & \\
\hline DQB1*06 & 55.7 & 44.4 & 0.2 & & & \\
\hline DQA1*01 & 75.4 & 74.6 & 1.0 & & & \\
\hline DQA $1 * 02$ & 29.5 & 30.2 & 1.0 & & & \\
\hline DQA1*03 & 14.7 & 22.2 & 0.3 & & & \\
\hline DQA1*04 & 3.3 & 1.6 & 0.6 & & & \\
\hline DQA1*05 & 37.7 & 50.8 & 1.1 & & & \\
\hline DQA1*06 & 0 & 1.6 & 0.2 & & & \\
\hline
\end{tabular}

${ }^{a}$ Pc value of $<0.05$ is considered significant. $\mathrm{N}-$ number of cases.

\section{RBC antibody testing techniques}

We identified clinically relevant RBC antibodies by routine indirect antiglobulin testing in a low ionic strength solution using a gel microcolumn hemagglutination technique (DG Gel ${ }^{\circledR}$; Grifols, Spain) with an 11-cell diagnostic panel of red blood cells (Identisera Diana; Grifols, Spain). We used an in-house adsorption tech- nique to resolve the anti-C+D mixture of antibodies from anti-G in pregnant women.

DNA extraction and HLA genotyping methods

DNA was isolated from blood samples using a spin column method (High Pure PCR Template Preparation Kit; Roche Diagnostics GmbH, Germany) or an automated technique (Maxwell 16 Blood DNA Purification Kit; Promega Corp., WI, USA). After obtaining an informed consent from the patients, HLA genotyping was performed. In the cohort of multi-responders, HLA genotyping of the -DRB1 and -DQB1/DQA1 loci was performed by polymerase chain reaction with sequence-specific oligoprobes (PCR-SSO), using Luminex technology with an analysis platform of a microsphere-based assay (LABType SSO DRB1 and LABType SSO DQA1/DQB1; One Lambda, CA, USA).

\section{Statistical analyses}

The phenotypic frequencies of the HLA-DRB1, HLA-DQB1, and HLA-DQA1 variants were compared between $\mathrm{RhD}$-positive and RhD-negative patient subgroups, and between RhD-positive patients with the DCCee phenotype and RhD-positive patients with different RhD phenotypes (i.e., DCcee, DCcEe, DccEe, and DccEE). These comparisons were performed using Pearson's test or Fisher's exact test (when there were few patients in a subgroup). We calculated the odds ratio (OR) with a $95 \%$ confidence interval (CI), and the p value. Nominal p values of $<0.05$ were corrected for multiple comparisons using the Bonferroni method. Between-group differences with corrected p values $(P c)$ of $<0.05$ were considered statistically significant.

\section{Results}

Our heterogeneous cohort included 124 alloimmunized patients (female-to-male [F/M] ratio, 2.76) with at least two RBC alloantibody specificities (multi-responders). In these patients, we detected 19 different RBC antibodies (Tab. 1). Of the 124 individuals, 72 (69 \%; F/M ratio, 2.4) underwent transfusion therapy at the University Hospital of Ostrava (median, 4 units; range, 1-48 units) and were tested within the follow-up period at our department, while 31 patients (F/M ratio, 1.7) with multiple antibody specificities had no transfusion 
Tab. 3. Comparisons of phenotypic frequencies of HLA-DRB1, HLA-DQB1, and HLA-DQA1 phenotypes $(n=30)$.

\begin{tabular}{|c|c|c|c|c|c|c|}
\hline $\begin{array}{l}\text { Allelic } \\
\text { groups }\end{array}$ & $\begin{array}{l}\text { Percentage of } \\
\text { Rh DCCee } \\
\text { phenotype }\end{array}$ & $\begin{array}{c}\text { Percentage of all } \\
\text { other } \mathrm{RhD}(+) \\
\text { phenotypes }\end{array}$ & $\mathrm{p}$ & OR & $95 \%$ CI & Pc value $^{a}$ \\
\hline DRB1*01 & 24.2 & 20.0 & 0.8 & & & \\
\hline DRB1*03 & 30.3 & 26.7 & 0.8 & & & \\
\hline DRB1*04 & 9.1 & 36.7 & 0.013 & 0.17 & $0.04-0.7$ & NS \\
\hline DRB1*07 & 24.2 & 36.7 & 0.4 & & & \\
\hline DRB1*08 & 0 & 6.7 & 0.2 & & & \\
\hline DRB1*09 & 0 & 0 & 1.0 & & & \\
\hline DRB1*10 & 0 & 3.3 & 0.5 & & & \\
\hline DRB1*11 & 21.2 & 6.7 & 0.2 & & & \\
\hline DRB1*12 & 0 & 3.3 & 0.1 & & & \\
\hline DRB1*13 & 45.5 & 3.3 & 0.0001 & 24 & 2.9-199 & 0.015 \\
\hline DRB1*14 & 3.0 & 10.0 & 0.3 & & & \\
\hline DRB1*15 & 27.3 & 23.3 & 0.8 & & & \\
\hline DRB1*16 & 9.1 & 6.7 & 0.4 & & & \\
\hline DQB1*02 & 42.4 & 50.0 & 0.8 & & & \\
\hline DQB1*03 & 39.4 & 56.7 & 0.3 & & & \\
\hline DQB1*04 & 0 & 3.3 & 0.5 & & & \\
\hline DQB1*05 & 39.4 & 40.0 & 1.0 & & & \\
\hline DQB1*06 & 63.6 & 23.3 & 0.002 & 5.8 & $1.9-17.3$ & 0.01 \\
\hline DQA1*01 & 93.9 & 53.3 & 0.0003 & 13.5 & $2.7-67.2$ & 0.002 \\
\hline DQA1*02 & 24.2 & 36.7 & 0.5 & & & \\
\hline DQA1*03 & 9.1 & 36.7 & 0.014 & 0.17 & $0.04-0.7$ & NS \\
\hline DQA1*04 & 0 & 3.3 & 0.5 & & & \\
\hline DQA1*05 & 54.5 & 46.7 & 0.6 & & & \\
\hline DQA1*06 & 0 & 3.3 & 0.5 & & & \\
\hline
\end{tabular}

${ }^{a}$ Pc value of $<0.05$ is considered significant. NS - not significant. $\mathrm{N}-$ number of cases. variants among multi-responders with the DCCee phenotype $(n=33)$ and all other $D$-positive

viduals was 1.07 among females and 0.94 among males. As expected, the combination of anti-C+D antibodies was found only in D-negative individuals (F/M ratio, 2.6, including two pregnant women). In contrast, the combination of anti-E+other specificities (any of c/Cw/C/D/K/Le $/ \mathrm{Fy}^{\mathrm{a} /} / \mathrm{Jk}^{\mathrm{a}} / \mathrm{Jk}^{\mathrm{b}} / \mathrm{M}$, etc.), occurred in patients with both major RhD phenotypes. The frequency of individuals carrying anti-C+D antibodies reached $75.4 \%$, representing the most common alloantibody combination in RhD-negative individuals. The combination of anti-E+other specificities was detected in 61 cases (49.2\%) among all 124 multi-responders, and in 44 cases (69.8\%) among the $63 \mathrm{RhD}$ positive individuals ( $\mathrm{F} / \mathrm{M}$ ratio, 2.8, including 10 pregnant females). Table 1 shows all observed RBC antibody combinations.

Further exploration of a subgroup of 20 pregnant women with multiple RBC alloantibodies revealed $8 \mathrm{RhD}$-negative and 12 RhD-positive phenotypes. Among pregnant women, the anti-E+c antibody combination was detected in 5 cases, anti-E+Cw in 4 cases, anti-D+E, C+G, C+D in 2 cases each, and other combinations in 1 case each (i.e., $\mathrm{E}+\mathrm{Le}^{\mathrm{a}}, \mathrm{D}+\mathrm{Le}^{\mathrm{a}}, \mathrm{C}+\mathrm{E}, \mathrm{e}+\mathrm{C}$ and $\left.\mathrm{c}+\mathrm{K}\right)$.

history at our hospital. The cohort included 91 women, of whom 20 (22\%) had alloantibody-complicated pregnancies. The mean age was 66.3 years (range, 42-90 years) among the 33 males, 69 years (range, 39-91 years) among the 71 females, excluding the pregnant women, who had the average age of 31.9 years (range, 22-43 years).

Analysis of multiple RBC alloantibody profiles and RhD phenotypes revealed two main patterns. As expected, antibody combinations including anti-D were associated with a RhD-negative phenotype, while the combinations including anti-E were common for both major RhD phenotypes. However, anti-E was formed by D-negative individuals at a lower frequency than by D-positive individuals (27.9\% vs $72.1 \%$ ). Anti-D was more commonly present in combination with some common specificities, including $\mathrm{D}+\mathrm{C}$ (75.4 \%) and D+E (19.7\%), and much less frequently combined with others, e.g., D+K and D+Le ${ }^{\mathrm{a}}$ (3.3\%). The D-negative group exhibited a high F/M ratio (2.8), necessitating that we consider pregnancy-mediated RBC alloimmunization, in addition to transfusion. Several antibody combinations were apparent only in RhDpositive cases, including $\mathrm{E}+\mathrm{c}(30.2 \%), \mathrm{e}+\mathrm{C}(12.7 \%), \mathrm{E}+$ other (non-Rh blood group system) specificities (17.5\%), and $\mathrm{K}+\mathrm{C} / \mathrm{Cw} / \mathrm{C}$ (6.3\%). Other specificities occurred in both RhD phenotypes but predominantly in $\mathrm{RhD}$-positive cases. A combination of $\mathrm{E}+\mathrm{CW}$ antibodies was found in $11.3 \%$ of individuals, and a mixture of non-E and non-D antibodies occurred in $10.5 \%$ of cases.

The RhD-negative phenotype was observed in $49.2 \%$ of multiresponders, and the ratio of $\mathrm{RhD}$-positive to $\mathrm{RhD}$-negative indi-
The proportion of the RhD-negative phenotype was substantially higher in our group of multi-responders (49.2\%) compared to that reported in Caucasian populations (range, 12-18\%) and HLA-DRB1*15 has previously been described as susceptibility marker for RBC multiple alloimmunization, therefore, we performed a sub-analysis of the HLA-Class II variants according to the patients' RhD status. Importantly, in our group of multiresponders, we found a significantly higher phenotypic frequency (proportion of carriers) of HLA-DRB1*15 in D-negative patients than in D-positive individuals ( $\mathrm{PC}_{\mathrm{C}}=0.045$ ). This finding was in agreement with analyses of the relationship between HLA-DRB1 and combinations of alloantibodies, which revealed 28 (60.9\%) DRB1*15 carriers among 46 individuals forming anti-C+D alloantibodies, but only 3 DRB1*15-positive subjects exhibiting other alloantibody combinations. Table 2 presents the phenotypic frequencies of HLA-DRB1, HLA-DQB1, and HLA-DQA1 in Dnegative and D-positive multi-responders.

Among the D-positive multi-responders, we performed a more detailed investigation of the DCCee phenotype, which was the most prevalent phenotype among D-positive cases (52.4 \%; F/M ratio, 2.0). Notably, the individuals with this phenotype could theoretically form combinations of anti-E+c alloantibodies. In fact, among 33 patients with the DCCee phenotype, 20 cases (60.6\%) formed the anti-E+c combination. We then compared the distribution of HLA-Class II variants between the patients with the DCCee phenotype and all other RhD-positive phenotypes. Individuals with the DCCee phenotype exhibited a significantly higher frequency 
of HLA-DRB1*13 (Pc = 0.015), and further association of HLA alleles in linkage disequilibrium (HLA-DQB1*06, $P_{\mathrm{C}}=0.01$; HLA-DQA1*01, Pc $=0.002)($ Tab. 3).

\section{Discussion}

In this study, we investigated the distribution of HLA-Class II variants within subgroups of multi-responders to RBC alloantigens, with the aim of elucidating associations between HLA-Class II alleles, Rh phenotypes, and RBC alloimmunization. In the previous studies, HLA-DRB1*15 has been associated with multiple RBC antibody responsiveness (13-15) and with anti-Fy alloimmunization $(10,11,16)$. Here we found that the DRB1*15 variant was twice as frequent in multi-responders with the D-negative phenotype as in RhD-positive multi-responders. In other words, it appears that DRB1*15 was associated with multiple alloimmunization (namely anti-C+D), particularly among D-negative individuals. Additionally, HLA-DRB1*13 and the variants in LD with DRB1*13 (namely HLA-DQB1*06 and HLA-DQA1*01) were associated with the DCCee phenotype, but not with other D-positive phenotypes (i.e., DCcee, DCcEe, DccEe, and DccEE).

In this analysis, we applied a new approach for stratifying the genetic risk for multiple alloimmunization conferred by HLA variants with the implementation of major RBC phenotypes (antigens), since an absence of certain RBC antigen(s) represents a necessary condition for the patients to be immunized against such antigen(s) and to develop alloantibodies. These findings are in concordance with our previous study, in which we found that the DRB1*15 allele was associated with $\mathrm{C}+\mathrm{D}$ alloantibodies, and the HLA-DRB $1 * 13$ allele (HLA-DQB $1 * 06$ and HLA-DQA $1 * 01$ ) was associated with $\mathrm{E}+\mathrm{c}$ alloantibodies (14). Multiple antibody formation seems to be less dependent on the presence of antigenspecific HLA-Class II restriction genes, and more dependent on the immunization to a first and often single antibody. Moreover, immune priming to one RBC alloantigen can directly increase a humoral response to additional RBC alloantigens following subsequent transfusion (17).

In our group of multi-responders, 112 individuals (90.3\%) were negative for $\mathrm{RhE}$ antigen (ratio of RhD-positive to RhD-negative, 0.84 ), and this group could be immunized by allogeneic RhE antigen and form anti-E antibodies. In fact, we found anti-E antibodies (together with other specificities) in 61 cases (54.5\%), suggesting that anti-E immunization was a frequent complication of transfusion in both RhD groups. Therefore, we additionally analysed the distribution of HLA-DRB1 variants according to the RhE-positive and RhE-negative phenotypes. However, we found no significant changes in the distribution of HLA-Class II variants between the RhE-negative and RhE-positive cases, possibly due to the small size of the RhE-positive subgroup.

Long-term evidence shows that patients with the D-negative phenotype are obviously more prone to RBC alloimmunization. In the recent report from the large Recipient Epidemiology and Donor Evaluation Study (REDS-III), the proportion of RBC alloimmunized patients was $45 \%$ higher among D-negative patients than in D-positive patients (18). This finding is in agreement with the higher frequency of D-negative phenotypes (49.2 \%) among the multi-responders in our study. Overall, accumulating evidence indicates that transfused patients with the D-negative phenotype carry a higher risk of developing multispecific RBC alloantibodies.

With regards to the previous reports that HLA-DRB1*15 confers susceptibility to RBC alloimmunization, Sippert et al compared alloimmunized and non-alloimmunized patients, and identified a significant relationship between $\mathrm{Rh}$ alloimmunization and DRB1*15 (19). Similarly, Moritz et al reported that HLADRB1*15 was associated with an increased risk of Rh alloimmunization (20). Several studies demonstrated that HLA-DRB1*15 was frequently present in multiple RBC antibody responders and might define a high-responding phenotype (13-15). The other recent investigations confirmed that the frequency of HLA-DRB1*15 was higher in the multi-responder group than in non-responders, which support the idea that HLA typing before transfusion could be helpful in reducing alloimmunization in chronically transfused patients $(21,22)$.

Despite growing data supporting a relationship between HLA and RBC alloimmunization, the exact mechanism underlying the association between DRB1*15 and immunization against multiple alloantigens is unknown. Some authors speculate that these findings should not be considered an evidence of more efficient antigen presentation, but rather indications that DRB1*15 is part of a susceptible and immune response "amplifier" haplotype, e.g., for certain cytokine profiles (15).

\section{Conclusions}

In conclusion, our present results suggest that the carriage of HLA-DRB1*15 in the D-negative phenotype, and of HLADRB1*13 (together with DQB1*06 and DQA1*01) in the DCCee phenotype, may further increase susceptibility to multi-responsiveness to RBC alloantigens. These findings may contribute to the identification of patients at higher risk of multiple RBC alloimmunization. For such patients, an extended RBC antigen matching (D and E antigens) could be considered, especially in transfusion-dependent D-negative patients possessing HLADRB1*15 and/or those patients, who are E-negative with the HLA-DRB1*13 variant.

\section{References}

1. Davenport RD. Pathophysiology of hemolytic transfusion reactions. Semin Hematol 2005; 42: 165-168.

2. Higgins JM, Sloan SR. Stochastic modeling of human RBC alloimmunization: evidence for a distinct population of immunologic responders. Blood 2008; 112: 2546-2553.

3. Zalpuri S, Zwaginga JJ, le Cessie S, Elshuis J, Schoenewille H, van der Bom JG. Redblood-cell alloimmunization and number of red bloodcell transfusions. Vox Sang 2012; 102: 144-149.

4. Drasar E, Igbineweka N, Vasavda N, Free M, Awogbade M, Allman M, Mijovic A, Thein SL. Blood transfusion usage among adults with sickle cell disease - A single institution experience over ten years. Br J Haematol 2011; 152: 766-770. 
5. Tormey CA, Fisk J, Stack G. Red blood cell alloantibody frequency, specificity, and properties in a population of male military veterans. Transfusion 2008; 48: 2069-2076.

6. Yazdanbakhsh K, Ware RE, Noizat-Pirenne F. Red blood cell alloimmunization in sickle cell disease: Pathophysiology, risk factors, and transfusion management. Blood 2012; 120: 528-537.

7. Ameen R, Al-Shemmari S, Al-Humood S, Chowdhury RI, Al-Eyaadi O, Al-Bashir A. RBC alloimmunization and autoimmunization among transfusion-dependent Arab thalassemia patients. Transfusion 2003; 43: 1604-1610.

8. Wang LY, Liang DC, Liu HC, Chang FC, Wang CL, Chan YS. Alloimmunization among patients with transfusion-dependent thalassemia in Taiwan. Transfus Med 2006; 16: 200-203.

9. Reviron D, Dettori I, Ferrera V, Legrand D, Touinssi M, Mercier P, de Micco P, Chiaroni J. HLA-DRB1 alleles and Jk(a) immunization. Transfusion 2005; 45 (6): 956-959.

10. Noizat-Pirenne F, Tournamille Ch, Bierling $P$, Roudot-Thoraval F, Le Penne PY, Rouger P, Ansart-Pirenne $\mathbf{H}$. Relative immunogenicity of $\mathrm{Fy}^{\mathrm{a}}$ and $\mathrm{K}$ antigens in a Caucasian population, based on HLA class II restriction analysis. Transfusion 2006; 46: 1328-1333.

11. Picard C, Frassati C, Basire A, Buhler S, Galicher V, Ferrera V, Reviron D, Zappitelli JP, Bailly P, Chiaroni J. Positive association of DRB1*04 and DRB1*15 alleles with Fy ${ }^{\mathrm{a}}$ immunization in a Southern European population. Transfusion 2009; 49(11): 2412-2417.

12. Chiaroni J, Dettori I, Ferrera V, Legrand D, Touinssi M, Mercier P, de Micco P, Reviron D. HLA-DRB1 polymorphism is associated with Kell immunization. Br J Haematol 2006; 132(3): 374-378.

13. Schonewille H, Doxiadis I I N, Levering W H B M, Roelen D L, Claas F H J, Brand A. HLA-DRB1 associations in individuals with single and multiple clinically relevant red blood cell antibodies. Transfusion 2014; 54: 1971-1978.

14. Maluskova A, Mrazek F, Pauliskova M, Kovarova P, Koristka M, Jindra P, Cermakova Z. Association of HLA-DRB1 and HLA-DQB1 with red-blood-cell alloimmunization in the Czech population. Vox Sang 2017; 112: 156-162.
15. Verduin EP, Brand A, van de Watering LM, Roelen DL, Kanhai HHH, Doxiadis IIN, Claas FHJ, Schonewille H. The HLA-DRB1*15 phenotype is associated with multiple red blood cell and HLA antibody responsiveness. Transfusion 2016; 56: 1849-1856.

16. Raos M, Zunec R, Mocibob B, Gojceta K, Lukic M, Cepulic BG. Susceptible and protective HLA-DR and HLA-DQX alleles for Fy ${ }^{\mathrm{a}}$ alloimmunization in the Croatian population. Transfusion 2019; 59: 1118-1124.

17. Patel SR, Bennett A, Girard-Pierce K, Maiker CL, Chonat A, Arthur CM, Zerra PE, Mener A, Stonewell SR. Recipient priming to one RBC alloantigen directly enhanced subsequent alloimmunization in mice. Blood Advances 2018; 2 (2): 105-115.

18. Karafin MS, Westlake M, Hauser RG, Tormey CA, Norris PJ, Roubinian NH, Wu Y, Triulzi DJ, Kleinman S, Hendrickson JE. NHLBI Recipient Epidemiology and Donor Evaluation Study-III (REDS-III). Risk factors for red blood cell alloimmunization in the Recipient Epidemiology and Donor Evaluation Study (REDS-III) database. Br J Haematol 2018; 181 (5): 672-681.

19. Sippert EA, Visentainer JEL, Alves HV, Rodrigues C, Gilli SC, Addas-Carvalho M, Saad STO, Costa FF, Castilho L. Red blood cell alloimmunization in patients with sickle cell disease: correlation with HLA and cytokine gene polymorphisms. Transfusion 2017; 57: 379-389.

20. Moritz E, Souza CP, Cruz B, et al. HLA-DRB1*12 and HLADRB1*15 are associated with antibody response to RHD antigen in Brazilians. Vox Sang 2015; 109 Suppl 1: 376.

21. Darvishi P, Sharif Z, Azarkievan A, Akbari A, Pourfathollah AA 2018. HLA-DRB1*15: 03 and HLA-DB1*11: useful predictive alleles for alloantibody production in thalassemia patients. Transfusion Med 2019; 29 (3): 179-184.

22. Ebrahimi M, Dayer D, Jalalifar MA, Keikhaei B, Tahan Nejadi Asadi Z. Association between HLA-DRB1*01 and HLA-DRB1*15 with alloimmunization in transfusion-dependent patient wit thalassemia. Transfusion Med 2020; 1-6.

Received September 30, 2020. Accepted October 21, 2020 